\title{
High predictability for identifying Lynch syndrome via microsatellite instability testing or immunohistochemistry in all Lynch-associated tumor types
}

\author{
John M. Carethers \\ Division of Gastroenterology, Department of Internal Medicine and Department of Human Genetics and Rogel Cancer Center, University of \\ Michigan, Ann Arbor, Michigan, USA \\ Correspondence to: John M. Carethers, MD. Division of Gastroenterology, Department of Internal Medicine, University of Michigan, 3100 Taubman \\ Center, 1500 E. Medical Center Drive, Ann Arbor, MI 48109-5368, USA. Email: jcarethe@umich.edu. \\ Comment on: Latham A, Srinivasan P, Kemel Y, et al. Microsatellite instability is associated with the presence of Lynch syndrome pan-cancer. J Clin \\ Oncol 2019;37:286-95.
}

Submitted Jul 21, 2019. Accepted for publication Aug 05, 2019.

doi: $10.21037 /$ tcr.2019.08.10

View this article at: http://dx.doi.org/10.21037/tcr.2019.08.10

Lynch syndrome is an inherited cancer-prone syndrome identified in $\sim 3 \%$ of all colorectal cancer (CRC) patients and $\sim 2 \%$ of all endometrial cancer (EC) patients (1-3). It is defined by identification of a germline mutation in one of the DNA mismatch repair (MMR) genes ( $M S H 2$, MLH1, MSH6, PMS2, EPCAM) whose proteins function to maintain replication fidelity of DNA prior to mitosis, and sets it apart from other hereditary non-polyposis colorectal cancer (HNPCC) syndromes such as Familial Colorectal Cancer Type $\mathrm{X}$ and Polymerase Proofreading Polyposis syndrome (3). Patients with Lynch syndrome can develop synchronous or metachronous cancers at younger ages than patients with the counterpart sporadic cancers. CRC in both sexes and EC in women are the most common tumors observed, with other gastrointestinal cancers (stomach, biliary, small intestine, pancreas), female reproductive tract cancers (ovary), urinary tract cancers (renal pelvis, ureter), and brain tumors (glioblastoma) in the spectrum of Lynch tumors (1-3). The Muir-Torre variant of Lynch syndrome adds specific skin tumors such keratoacanthomas and sebaceous neoplasms to the tumor spectrum. The prevalence of Lynch syndrome in the population is estimated to be 1 in 300; yet it is underdiagnosed due to several factors including (I) little or no pre-symptomatic findings prior to cancer in patients, (II) unawareness by clinicians and patients, and (III) inadequate family history of cancer knowledge transmitted to the healthcare provider $(1,3)$. Because surveillance of Lynch syndrome patients extends their survival as well as quality of life, it is important to identify all patients. Universal population screening for Lynch syndrome among patients who present with colorectal and EC, the two most prevalent cancers in patients with the syndrome, has identified additional but not all Lynch patients that would have otherwise been missed and enrolled them in cancer surveillance programs, and generated the opportunity to offer genetic testing to affected and unaffected relatives (1-3).

Lynch syndrome is one of five human conditions in which tumors manifest a defect in MMR function that can be detected by microsatellite instability (MSI) testing and altered MMR protein expression (Table 1) (4-6). In Lynch syndrome, the patient manifests a mono-allelic MMR gene germline mutation in all cells of their body, and within developing or established tumor cells, the second MMR gene allele is inactivated somatically (two hits) thereby completely abating the expression of either allele of the MMR gene. The complete loss of the MMR protein expression partially or fully inactivates MMR function, committing daughter cells to develop point mutations and slippage mistakes at DNA microsatellite sequences, the latter detected biochemically by PCR [or by next generation sequencing (NGS)] as MSI (2,4,7-9). The presence of MSI and the loss of MMR protein expression go hand-in-hand, with over a $94 \%$ concordance rate (7). Both tests individually are used to screen CRCs and ECs for potential Lynch syndrome patients $(2,3,6,9)$, with both a 
Table 1 Human DNA mismatch repair deficiency conditions and MMR gene and protein expression

\begin{tabular}{|c|c|c|}
\hline Condition & MMR inactivation mechanism & MMR protein expression \\
\hline $\begin{array}{l}\text { Lynch-like syndrome/double somatic } \\
\text { MMR inactivation }\end{array}$ & Bi-allelic somatic $M M R$ gene mutation & $\begin{array}{l}\text { Tumor loss of MSH2, MLH1, MSH6, or PMS2 } \\
\text { expression }\end{array}$ \\
\hline Sporadic MSI CRC & Bi-allelic somatic $M L H 1$ hypermethylation & Tumor loss of MLH1 (and PMS2) expression \\
\hline $\begin{array}{l}\text { Inflammation-associated microsatellite } \\
\text { alterations/EMAST }\end{array}$ & $\begin{array}{l}\text { Somatic nuclear-to-cytosolic MSH3 protein } \\
\text { shift }\end{array}$ & $\begin{array}{l}\text { Reduction and heterogeneity of nuclear } \mathrm{MSH} 3 \\
\text { expression }\end{array}$ \\
\hline
\end{tabular}

MMR, mismatch repair; EMAST, elevated microsatellite alterations at selected tetranucleotide repeats; CRC, colorectal cancer.

great improvement upon solely using the clinically-oriented Amsterdam criteria or Bethesda guidelines. CRCs and ECs that show MSI and/or MMR protein expression loss via immunohistochemistry (IHC) should inform the need for genetic counseling and genetic testing for a germline MMR gene mutation, the ultimate gold standard for characterizing Lynch syndrome families $(1,2,6)$.

Can we expand screening to identify more patients with Lynch syndrome? With the study by Latham et al. (10), the answer is yes. Using NGS examining 15,045 unique patients with cancer spanning 50 cancer types followed by IHC in specific tumors, Latham et al. was able to newly identify 103 Lynch patients (overall $0.7 \%$ ), with 53 of 326 (16.3\%) coming from patients with MSI-high tumors, 13 of $699(1.9 \%)$ coming from MSI-indeterminate tumors, and 37 of 14,020 (0.3\%) coming from microsatellite stable (MSS) tumors (10). CRC and EC represented 201 of 326 (62\%) of the MSI-high cohort while comprising only 1,351 of $15,045(9 \%)$ of the tumors (10). Of patients with identified Lynch syndrome and an MSI-high tumor, 33 of 66 (50\%) had a primary tumor other than CRC or EC, allowing the diagnosis of Lynch syndrome from the screening of other tumors rarely implicated in the syndrome, including adrenocortical cancer, mesothelioma, soft tissue sarcoma, pancreatic cancer, among others (Table 2) (10). IHC for MMR protein expression was concordant in 56 of 57 (98.2\%) of identified Lynch syndrome patients (10). Lynch syndrome was not diagnosed via screening of all tumor types. Most notably, none of 150 MSI-high from 2,371 breast cancers, none of the 46 MSI-high from 343 ovarian cancers, none of the 94 MSI-high from 1,952 lung cancers, and none of the 11 MSI-high from 458 renal cancers belonged to the identified Lynch syndrome patients
(Table 2) (10). Only 15 of 66 (22.7\%) of identified Lynch patients with MSI-high tumors had a prior malignancy history (10). In addition to Lynch syndrome, Latham et al. also detected 57 Lynch-like (double somatic MMR gene inactivation) patients (Table 1) out of 274 (20.8\%) non-Lynch MSI-high tumors (10). Thirty-seven of the 103 identified Lynch syndrome patients came from MSS tumors, with most showing germline mutation of MSH6 and PMS2, two lower penetrant MMR genes, in contrast to the majority of identified Lynch patients from MSI-high tumors showing MLH1 and MSH2 mutations (10). Overall, Latham et al. demonstrates with expansion of MSI and IHC testing to multiple cancers beyond CRCs and ECs that an additional $50 \%$ of Lynch syndrome patients can be newly identified.

There are a few other observations from Latham et al. First, the level of MSI varied among the various tumor types, with higher levels of MSI-high found among ureter, gastric and CRCs and lower levels found in ECs and brain tumors (10). MSI-high tumors are hypermutated due to the nature of the MMR defect, and contain hundreds of somatic mutations $(9,11)$. Variance in the degree of hypermutation might be reflective of (I) high penetrant versus low penetrant MMR gene inactivation, (II) dominant clone and sampling from the tumor which may be heterogeneous, and/ or (III) tumor cell doubling times to allow for more somatic mutation accumulation (12). Second, the race/ethnicity make-up of patients with MSI-high tumors was markedly lower for non-Hispanic blacks as compared to non-Hispanic white patients, consistent with prior population studies among sporadic CRC patients $(13,14)$. Third, Latham et al. only screened patient cancers. Other non-cancers, such as sebaceous neoplasms that can be a part of Lynch syndrome, 
Table 2 Lynch-associated cancers that may reveal a Lynch syndrome diagnosis (10)

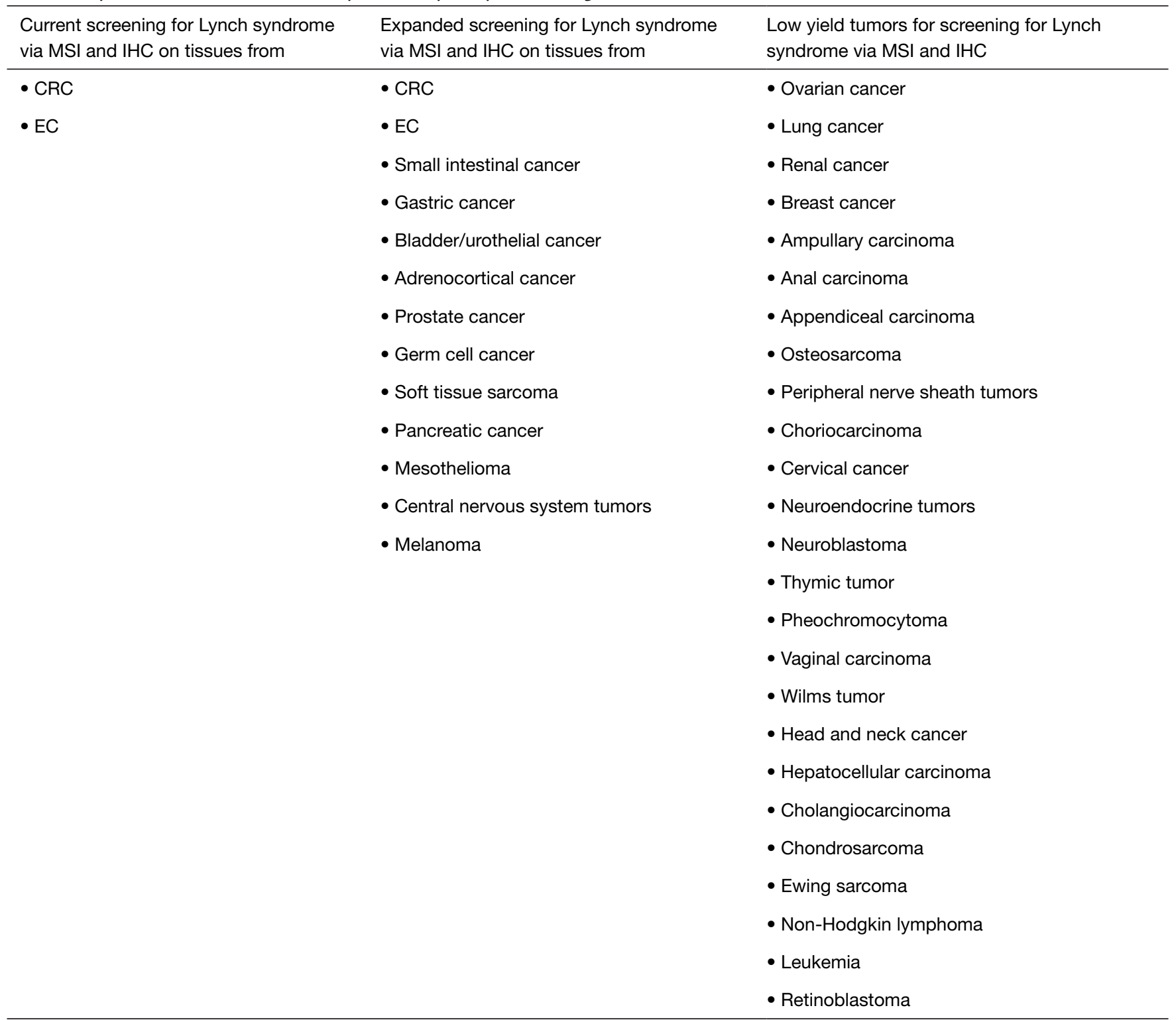

MSI, microsatellite instability testing; IHC, immunohistochemistry.

have shown loss of MMR protein expression that can subsequently identify germline MMR gene mutation (15). It is possible that screening specific non-cancers in addition to cancers might also improve the yield in identifying Lynch syndrome. And fourth, Latham et al. noted no identified Lynch patients from MSI-high breast cancers, but did identify 7 Lynch patients from among 2,371 (0.3\%) MSS breast cancers (10). Because these breast tumors lacked hypermutation and MSI, it is very likely that the breast tumor developed independently from the MMR germline mutation. Lynch syndrome patients can develop non-MSI tumors that are thought to be sporadic in addition to their MMR-deficient driven tumors, and breast cancer has been a controversial association within Lynch syndrome. Among all MSS cancers, Lynch syndrome was identified in $0.3 \%$, which is identical to the 1 in 300 population prevalence, strengthening the notion that the germline findings using NGS among MSS tumors is incidental and not causal.

Patients whose tumors show MSI-high, which includes all Lynch syndrome patients, may obtain a survival benefit 
with immune checkpoint blockade due to the hypermutated nature of the tumors and the generation of neoantigens from transcribed and subsequently translated genes that contain microsatellite coding sequences $(4,9,16)$. The U.S. Food and Drug Administration approved anti-PD-1 therapy for patients with MSI-high tumors, agnostic to the type of cancer. Thus, identifying Lynch syndrome patients is not only important for surveillance and prevention of future cancer, it affects the therapeutic options in identified patients that may develop a cancer. However, despite expansion to multiple tumor types to screen from and to identify Lynch syndrome, these various tumor types present in Lynch syndrome patients do not mean there is a uniform response to anti-PD-1 therapy. The extent of insertion/ deletion mutations (frameshifts at DNA microsatellites) within each tumor determines the level of PD-1 expression, and thereby the level of tumor cell response to anti-PD-1 therapy, and thereby the level of patient response to antiPD-1 therapy (17). Thus, the level of insertion/deletion mutational burden within each tumor determines that degree of potential response. Overall, the findings from Latham et al. broadens the catch and identification of Lynch syndrome patients for which surveillance and therapeutic options should improve outcomes.

\section{Acknowledgments}

Funding: The article was supported by the United States Public Health Service (NIH grant CA206010) and the A. Alfred Taubman Medical Research Institute of the University of Michigan. The funders had no role in study design, data collection and analysis, decision to publish, or preparation of the manuscript.

\section{Footnote}

Provenance and Peer Review: This article is commissioned and reviewed by the Academic Editor Jingping Wang (Department of Pathology, The First Affiliated Hospital of Bengbu Medical College, Bengbu Medical College, Bengbu, China).

Conflicts of Interest: The author has completed the ICMJE uniform disclosure form (available at http://dx.doi. org/10.21037/tcr.2019.08.10). The author has no conflicts of interest to declare.

Ethical Statement: The authors is accountable for all aspects of the work in ensuring that questions related to the accuracy or integrity of any part of the work are appropriately investigated and resolved.

Open Access Statement: This is an Open Access article distributed in accordance with the Creative Commons Attribution-NonCommercial-NoDerivs 4.0 International License (CC BY-NC-ND 4.0), which permits the noncommercial replication and distribution of the article with the strict proviso that no changes or edits are made and the original work is properly cited (including links to both the formal publication through the relevant DOI and the license). See: https://creativecommons.org/licenses/by-nc-nd/4.0/.

\section{References}

1. Stoffel EM, Boland CR. Genetics and genetic testing in hereditary colorectal cancer. Gastroenterology 2015;149:1191-203.

2. Carethers JM. DNA testing and molecular screening for colon cancer. Clin Gastroenterol Hepatol 2014;12:377-81.

3. Carethers JM, Stoffel EM. Lynch syndrome and Lynch syndrome mimics: the growing complex landscape of hereditary colon cancer. World J Gastroenterol 2015;21:9253-61.

4. Carethers JM. Microsatellite instability pathway and EMAST in colorectal cancer. Curr Colorectal Cancer Rep 2017;13:73-80.

5. Carethers JM. Hereditary, sporadic and metastatic colorectal cancers are commonly driven by specific spectrums of defective DNA mismatch repair components. Trans Am Clin Climatol Assoc 2016;127:81-97.

6. Carethers JM. Differentiating Lynch-like from Lynch syndrome. Gastroenterology 2014;146:602-4.

7. Boland CR, Thibodeau SN, Hamilton SR, et al. A National Cancer Institute Workshop on microsatellite instability for cancer detection and familial predisposition: Development of international criteria for the determination of microsatellite instability in colorectal cancer. Cancer Res 1998;58:5248-57.

8. Grady WM, Carethers JM. Genomic and epigenetic instability in colorectal cancer pathogenesis.

Gastroenterology 2008;135:1079-99.

9. Carethers JM, Jung BH. Genetics and genetic biomarkers in sporadic colorectal cancer. Gastroenterology 2015;149:1177-90.

10. Latham A, Srinivasan P, Kemel Y, et al. Microsatellite Instability Is Associated With the Presence of Lynch 
Syndrome Pan-Cancer. J Clin Oncol 2019;37:286-95.

11. Cancer Genome Atlas Network. Comprehensive molecular characterization of human colon and rectal cancer. Nature 2012;487:330-7.

12. Fearon ER, Carethers JM. Molecular subtyping of colorectal cancer: time to explore both intertumoral and intratumoral heterogeneity to evaluate patient outcome. Gastroenterology 2015;148:10-3.

13. Carethers JM, Murali B, Yang B, et al. Influence of race on microsatellite instability and CD8+ T cell infiltration in colon cancer. PLoS One 2014;9:e100461.

14. Ashktorab H, Ahuja S, Kannan L, et al. A meta-analysis of

Cite this article as: Carethers JM. High predictability for identifying Lynch syndrome via microsatellite instability testing or immunohistochemistry in all Lynch-associated tumor types. Transl Cancer Res 2019;8(Suppl 6):S559-S563. doi: 10.21037/ tcr.2019.08.10
MSI frequency and race in colorectal cancer. Oncotarget 2016;7:34546-57.

15. Everett JN, Raymond VM, Dandapani M, et al. Screening for germline mismatch repair mutations following diagnosis of sebaceous neoplasm. JAMA Dermatol 2014;150:1315-21.

16. Koi M, Carethers JM. The colorectal cancer immune microenvironment and approach to immunotherapies. Future Oncol 2017;13:1633-47.

17. Mandal R, Samstein RM, Lee KW, et al. Genetic diversity of tumors with mismatch repair deficiency influences antiPD-1 immunotherapy response. Science 2019;364:485-91. 